\title{
Probabilistic modelling of a rotational energy harvester
}

Journal of Intelligent Material Systems and Structures

I-9

(c) The Author(s) 2015

Reprints and permissions:

sagepub.co.uk/journalsPermissions.nav DOI: I0.II77// 045389XI5573343 jim.sagepub.com

\section{PL Green', M Hendijanizadeh ${ }^{2}$, L Simeone $^{2}$ and SJ Elliott ${ }^{2}$}

\begin{abstract}
Relatively recently, many researchers in the field of energy harvesting have focused on the concept of harvesting electrical energy from relatively large-amplitude, low-frequency vibrations (such as the movement caused by walking motion or ocean waves). This has led to the development of 'rotational energy harvesters' which, through the use of a rackand-pinion or a ball-screw, are able to convert low-frequency translational motion into high-frequency rotational motion. A disadvantage of many rotational energy harvesters is that, as a result of friction effects in the motion transfer mechanism, they can exhibit large parasitic losses. This results in nonlinear behaviour, which can be difficult to predict using physical-law-based models. In the current article a rotational energy harvester is built and, through using experimental data in combination with a Bayesian approach to system identification, is modelled in a probabilistic manner. It is then shown that the model can be used to make predictions which are both accurate and robust against modelling uncertainties.
\end{abstract}

\section{Keywords}

Bayesian inference, nonlinear dynamics, rotational energy harvesting

\section{Introduction}

Over the last decade the supposition that electrical energy can be harvested from ambient vibration sources has been the subject of a considerable amount of research. While much early work was focused on the development of small energy-harvesting devices which could be used to power microelectromechanical systems (MEMS) (such as small sensors which can be placed in hostile environments), more attention is now being paid to the concept of using relatively large devices to harvest energy from large-amplitude and/or low-frequency excitations.

As an example, Rome et al. (2005) proposed using a suspended load backpack to harvest the kinetic energy created by walking motion. Interestingly, it was reported that relatively little additional metabolic energy was required to carry the suspended load backpack when compared to a rigid backpack - it was hypothesised that this may be due to alterations in the biomechanics of walking which were induced by carrying the energy harvester. This led to the work of Papatheou and Sims (2012) where a hardware in-theloop energy-harvesting backpack was developed such that, by being able to easily vary different settings (such as stiffness and damping coefficients), a thorough investigation into the effect of the device on the human gait could be conducted: see Papatheou et al. (2012) for preliminary results. Additionally, work has also focused on whether, when harvesting energy from walking motion, the deliberate introduction of nonlinearities can be used to enhance energy harvester performance (Green et al., 2013).

Much research has also been focused on the possibility of using relatively large devices to harvest energy from the movement caused by ocean waves. Brown et al. (2007) investigated using energy harvesters to power ocean exploration robots. Deciding to reject solar panels and wind turbines in favour of vibrational energy harvesters (as they are potentially able to operate in all weather conditions and require no external machinery), a device was created which was able to fit inside an autonomous underwater glider. Matsuoka et al. (2002) used a combination of theoretical and experimental analysis to test various types of 'wave energy conversion systems' while, through experimental testing of a small-scale prototype, Agamloh et al. (2008)

\footnotetext{
'Department of Mechanical Engineering, University of Sheffield, Sheffield, UK; Institute for Risk and Uncertainty, University of Liverpool, UK ${ }^{2}$ ISVR, University of Southampton, Southampton, UK
}

\section{Corresponding author:}

PL Green, Department of Mechanical Engineering, University of Sheffield, Mappin Street, Sheffield, SI 3JD, United Kingdom.

Email: p.l.green@sheffield.ac.uk 
developed a device which was able to generate up to $69 \mathrm{~W}$ of power from wave motion.

In Choi et al. (2009) an energy harvester was developed which was designed to power an electrorheological automotive shock absorber. It was reported that the resulting device was compact enough to be attached to a real vehicle, and that its voltage output was large enough to obtain the required damping forces in the shock absorber. In subsequent work by Li et al. (2013) a device was constructed that was also designed to harvest energy from vehicle shock absorbers and, in a series of road tests, was able to generate an average power output of between 3.3 and $19.2 \mathrm{~W}$ (depending on vehicle speed).

With the low-frequency nature of the aforementioned applications in mind, many researchers have elected to develop energy harvesters which are able to convert low-frequency translational motion into highfrequency rotational motion (which is then used to generate electrical energy via an electromagnetic coupling). Of the works mentioned thus far, this conversion from translational to rotational motion has been achieved via either a rack-and-pinion (Choi et al., 2009; Li et al., 2013; Rome et al., 2005) or a ball-screw (Agamloh et al., 2008; Brown et al., 2007; Cassidy et al., 2011; Matsuoka et al., 2002). Further advantages of such a device were demonstrated in Hendijanizadeh et al. (2013), where the efficiencies of displacementconstrained translational and rotational energy harvesters were compared. It was found that the electromagnetic coupling coefficient of rotational energy harvesters scaled with device size at a greater rate than translational systems (thus making rotational energy harvesters more favourable in situations where relatively large devices are required).

Of particular interest here is the work of Cassidy et al. (2011) where a detailed study of a ball-screw rotational energy harvester was conducted. In their work the authors noticed that the dynamics of their device was strongly influenced by friction present in the ballscrew mechanism as well as other, hysteretic, behaviour. Using Coulomb damping to account for friction effects, a physical-law-based model of the device was developed. The parameters of the model were then estimated using a least-squares method while, to access parameter confidence, approximations of the parameter covariance matrix were also made.

The primary aim of the current paper is to build on the work of Cassidy et al. (2011) and conduct system identification of a rotational energy harvester (also with a ball-screw mechanism) using a probabilistic, Bayesian approach. To that end the experimental results from a series of tests of a rotational energy harvester have been analysed using Markov chain Monte Carlo (MCMC) methods, thus allowing the relative probability of two competing model structures - both of which feature a difference physical-law-based friction model - as well as

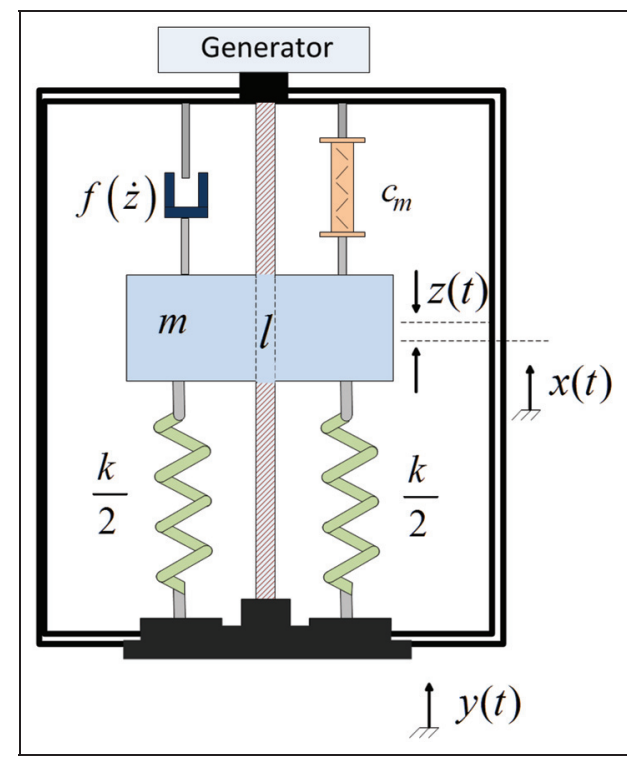

Figure I. Schematic of rotational energy harvester.

the probability of the parameters within each model to be assessed. The result is a probabilistic model ${ }^{1}$ of the energy harvester which can be used to make predictions about device performance which are robust against uncertainties in the modelling process (thus allowing one to probabilistically analyse the response of the device to various ambient excitations).

\section{Device description}

A schematic of the device in question is shown in Figure 1. It is comprised of a sprung mass coupled to an electrical generator using a ball-screw. The ballscrew converts the translational movement of the oscillating mass to rotatory motion which drives a brushless generator (see Hendijanizadeh, 2014, and Simeone et al., 2014, for a detailed description). The device was $25 \mathrm{~cm}$ wide and $110 \mathrm{~cm}$ tall.

Defining $l$ as the ball-screw lead, $c_{m}$ as mechanical damping, $k$ as spring stiffness, $m$ as the oscillating mass and $J$ as the moment of inertia of the system allows one to write the equation of motion of the device as

$$
M \ddot{z}+b_{m} \dot{z}+k z+f(\dot{z})=-m \ddot{y}, \quad z=x-y
$$

where $x$ is the displacement of the mass, $y$ is the displacement of the base

$$
\begin{gathered}
M=m+J\left(\frac{2 \pi}{l}\right)^{2} \\
b_{m}=\left(\frac{2 \pi}{l}\right)^{2} c_{m}
\end{gathered}
$$

and $f(\dot{z})$ is a friction model which has yet to be determined. 


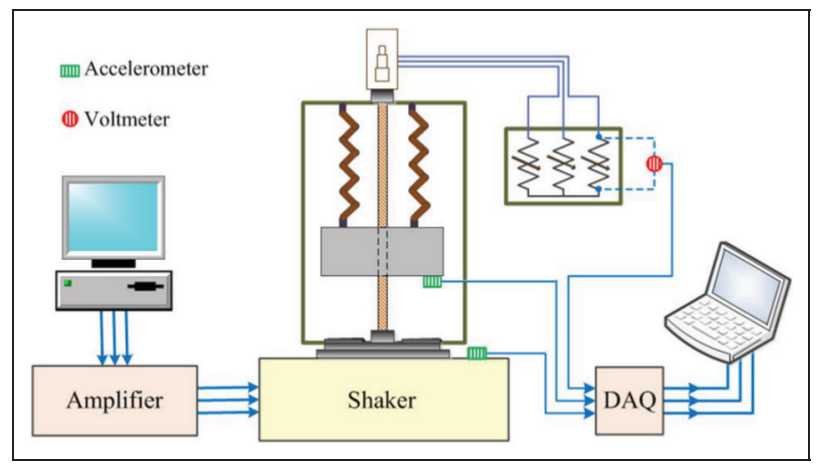

Figure 2. Schematic of experimental setup.

\section{Experiment}

Figure 2 shows a schematic of the experimental setup used to test the manufactured energy harvester. In this setup the harvester was mounted on a vertical electrohydraulic shaker and the generator terminals were connected to three variable resistors with a star configuration. Two MEMS accelerometers with a sensitivity of $800 \mathrm{mV} / \mathrm{g}$ and a dynamic range of $\pm 5 \mathrm{~g}$ were attached to the oscillating mass and the shaker. A voltmeter sensor was used to measure the voltage across the generator terminals, that is, the load resistance. The movement of the shaker was controlled by a PC through an amplifier model FE-376-IPF from FlydeSignal Ltd. The voltage output signal and the acceleration of the mass and shaker were captured by a National Instruments data acquisition (Daq) system with a sampling frequency of $256 \mathrm{~Hz}$. Figure 3 shows the actual implementation of the test rig, including the energy harvester, shaker and variable resistors.

In the current article the aim is to establish a probabilistic model of the mechanical portion of the device and, as such, all of the tests shown here were conducted under open circuit conditions. The excitation was Gaussian white noise, filtered by a low-pass filter with a cut-off frequency of $15 \mathrm{~Hz}$. The data used for system identification consisted of 2000 points of base acceleration time history $\ddot{y}$ (used as an input to the model) as well as 2000 points of relative acceleration $\ddot{z}$. During testing it was noticed that the relative acceleration measurements contained high-frequency components which arose as a result of a rocking mode of the oscillating mass. Given prior knowledge of the system (described further in 'Initial modelling'), the natural frequency of the system was expected to be less than $10 \mathrm{~Hz}$. Upon studying the frequency content of the data (Figure 4), it was clear that the rocking mode of the mass was causing oscillations between 30 and $80 \mathrm{~Hz}$. Consequently, before it was used as training data, the data was passed through a low-pass filter with a cut-off frequency of $20 \mathrm{~Hz}$. The power spectral density (PSD) and time history of the unfiltered and filtered data are shown in Figures 4 and 5 respectively.

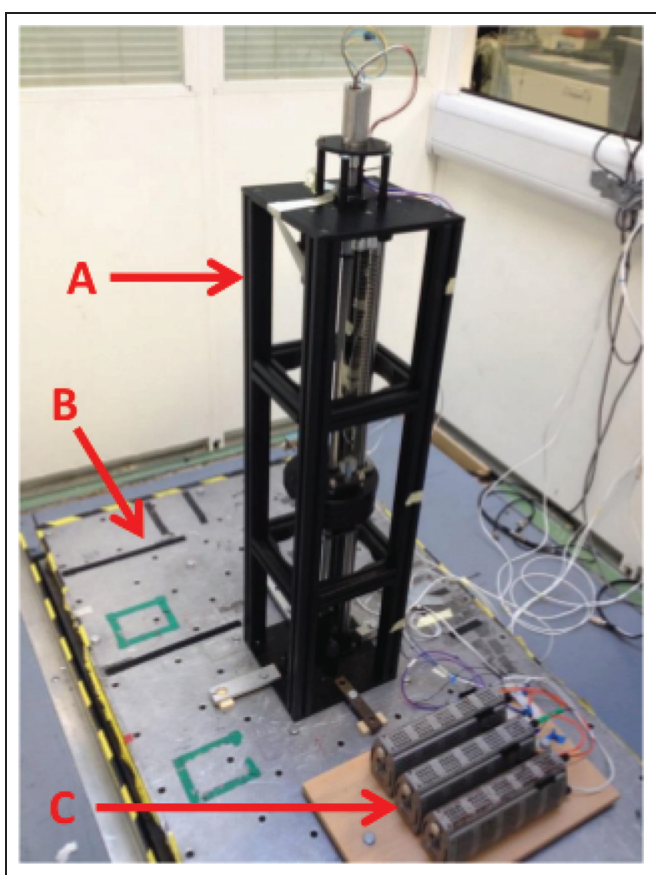

Figure 3. Actual implementation of energy harvester. A: Energy harvester device; B: shaker; C: variable resistors.

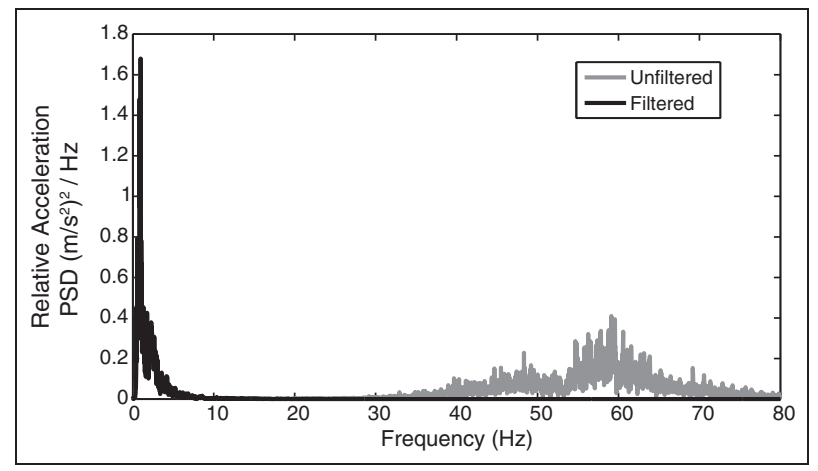

Figure 4. Filtered and unfiltered relative acceleration PSD.

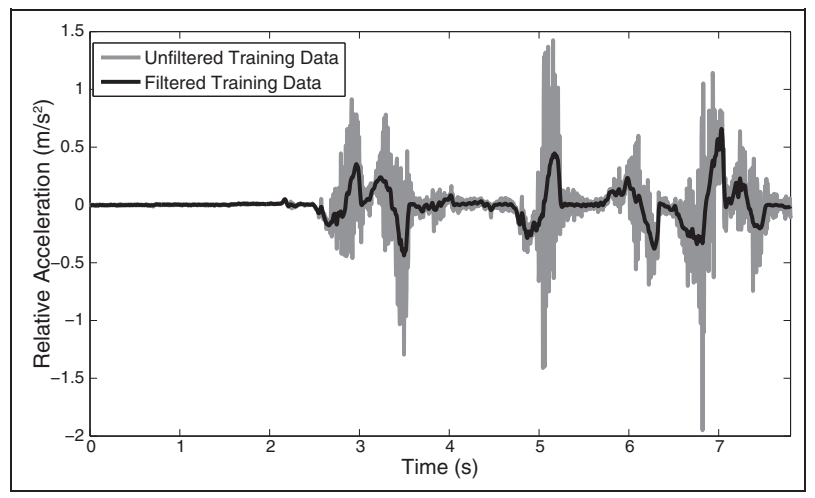

Figure 5. Filtered and unfiltered relative acceleration time history used as training data. 
To give a more quantitative impression of the energy harvester performance, the amplitudes of the excitations used during testing were compared with the amplitudes that the device may experience when excited by wave motion. To that end, the vertical acceleration of a boat (a double hull catamaran weighing approximately 3.5 tonnes) was measured while sailing in the English Channel. In this particular case the maximum acceleration recorded was around $6 \mathrm{~m} / \mathrm{s}^{2}$ while the maximum acceleration used during testing was roughly $4 \mathrm{~m} / \mathrm{s}^{2}$, confirming that the base acceleration used during testing was similar to the amplitude of excitation that the device would be subjected to if it were used to harvest energy from wave motion (although this is obviously rather dependent on weather conditions).

\section{Initial modelling}

Before embarking on Bayesian system identification, several tests were performed to produce prior estimates of the model parameters. To estimate the static Coulomb friction $\left(F_{c}\right)$ present in the device, the mass was displaced by an amount $x_{1}$ downwards such that, as the mass was held in equilibrium, it follows that

$$
m g+F_{c}=k x_{1}
$$

Repeating this process but displacing the mass by the amount $x_{2}$ upwards and noting that, to be in equilibrium

$$
m g=k x_{2}+F_{c}
$$

it follows that $2 F_{c} \approx k\left(x_{2}-x_{1}\right)$. Using the estimated value of spring stiffness $(250 \mathrm{~N} / \mathrm{m})$ and finding that $x_{2}$ $-x_{1}=0.067 \mathrm{~m}, F_{c}=8.4 \mathrm{~N}$ was used as a prior estimate for the static friction coefficient of the system. Subsequently, using a simple Coulomb damping model (such that $f(\dot{z})=F_{c} \operatorname{sgn}(\dot{z})$ ), the viscous damping term $c_{m}$ was estimated by fitting the frequency response of the model to the frequency response of the training data (Simeone et al., 2014). All of the resulting parameter estimates are shown in Table 1.

\section{A Bayesian approach}

The task is now to use the experimental data $D$, as well as the prior knowledge described in the previous section, to infer an appropriate model structure $M$ as well as the vector of parameters $\boldsymbol{\theta} \in \Theta \subset \mathbb{R}^{N_{\boldsymbol{\theta}}}$ within $M$ which require estimation. Through the sequential application of Bayes' theorem, it is possible to write both levels of inference - parameter estimation and model selection - as
Table I. Initial parameter estimates.

\begin{tabular}{lcc}
\hline Parameter & Measured Value & Units \\
\hline$M$ & 12.8 & $\mathrm{~kg}$ \\
$m$ & 8 & $\mathrm{~kg}$ \\
$k$ & 250 & $\mathrm{~N} / \mathrm{m}$ \\
$F_{c}$ & 8.4 & $\mathrm{~N}$ \\
$c_{m}$ & 170 & $\mathrm{Ns} / \mathrm{m}$ \\
\hline & & \\
& $P(\boldsymbol{\theta} \mid D, M)=\frac{P(D \mid \boldsymbol{\theta}, M) P(\boldsymbol{\theta} \mid M)}{P(D \mid M)}$ &
\end{tabular}

and

$$
P(M \mid D)=\frac{P(D \mid M) P(M)}{P(D)}
$$

respectively. Equation (7) allows one to assess the relative probability of two competing model structures. This is a particularly important tool with regard to model selection because, as it can be shown, models are assigned higher probabilities if they are able to replicate the training data without being overly complex (see Muto and Beck, 2008, for a comprehensive discussion).

The first aim is to evaluate the "posterior parameter distribution' $P(\boldsymbol{\theta} \mid D, M)$ in equation (6)- the probability of parameter vector $\boldsymbol{\theta}$ given the data $D$ and a chosen model structure $M . P(\boldsymbol{\theta} \mid M)$ is known as the prior and is a user-defined probability distribution describing one's knowledge of the parameters before the data was known. $P(D \mid \boldsymbol{\theta}, M)$ is known as the likelihood: this describes the probability of witnessing the data $D$ given that one has chosen to model the system using the structure $M$ with parameters $\boldsymbol{\theta}$. In this case, relying on the central limit theorem, it is assumed that the probability of witnessing a single data point $\left(\ddot{z}_{i}\right)$ is given by

$$
P\left(\ddot{z}_{i} \mid \boldsymbol{\theta}, M\right)=N\left(\hat{\ddot{z}}_{i}(\boldsymbol{\theta}), \sigma\right)
$$

where $\hat{\ddot{z}}_{i}(\boldsymbol{\theta})$ is the relative acceleration predicted by the model and the likelihood standard deviation $\sigma$ is treated as an unknown parameter for which probabilistic estimates will also be realised. Furthermore, it is assumed that the probability of witnessing each data point is mutually independent such that, for two data points $\ddot{z}_{i}$ and $\ddot{z}_{j}$

$$
P\left(\ddot{z}_{i}, \ddot{z}_{j} \mid \boldsymbol{\theta}, M\right)=P\left(\ddot{z}_{i} \mid \boldsymbol{\theta}, M\right) P\left(\ddot{z}_{j} \mid \boldsymbol{\theta}, M\right)
$$

so that the likelihood takes the form

$$
P(D \mid \boldsymbol{\theta}, M)=\prod_{i=1}^{N} N\left(\hat{\ddot{z}}_{i}(\boldsymbol{\theta}), \sigma\right)
$$

where $N=2000$. For the interested reader, a more thorough discussion on the choice of likelihoods is given by Simoen et al. (2013). 
The denominator in equation (6) is a normalising constant given by

$$
P(D \mid M)=\int P(D \mid \boldsymbol{\theta}, M) P(\boldsymbol{\theta} \mid M) d \boldsymbol{\theta}
$$

which is usually intractable and too time-consuming to evaluate numerically (as a result of the curse of dimensionality).

To generate samples from the posterior parameter distribution without having to evaluate equation (11), it is now common practice to employ MCMC methods such as the well-known Metropolis algorithm (Metropolis et al., 1953). MCMC essentially involves the creation of an ergodic Markov chain whose stationary distribution is equal to some target $\pi(\boldsymbol{\theta})$, up to a constant of proportionality (see Doob, 1953, for a comprehensive discussion on the convergence of Markov chains). Choosing one's target as the posterior parameter distribution then, by allowing the Markov chain to converge to its stationary distribution, one can use MCMC to generate samples from a distribution proportional to $P(\boldsymbol{\theta} \mid D, M)$. In the current article the transitional MCMC algorithm (TMCMC) is employed (Ching and Chen, 2007) as it is not only capable of targeting distributions with complex geometries, but is also able to estimate equation (11) which, as a result, allows equation (7) to be estimated.

\section{Results}

\section{Linear model}

Starting with a relatively simple model, it was first hypothesised that the friction effects present in the system could be modelled by increasing the viscous damping coefficient. Consequently, what is shown here is essentially a 'Bayesian equivalent linearisation' where the equivalent linear system is learnt from data. Acting under the assumption that $M, m$ and $k$ were estimated with sufficient accuracy, the vector of parameters to be estimated in this case was $\boldsymbol{\theta}=\left\{c_{m}, \sigma\right\}$ (where, as described in the previous section, $\sigma$ is the standard deviation of the likelihood). A Gaussian distribution (with moments shown in Table 2), truncated at zero, was used as a prior.

As described in the previous section, TMCMC (Ching and Chen, 2007) was used to generate 1000 samples from the posterior parameter distribution. Figure 6 shows the resulting samples from the posterior while the mean posterior parameter values are given in Table 3. With regard to Figure 6 it is interesting to note that the mean value of $c_{m}$ is approximately three times larger than the value that was estimated in 'Initial modelling', confirming that the system is strongly nonlinear.

Running Monte Carlo simulations (where each simulation utilises parameters which, using MCMC, have been sampled from the posterior parameter
Table 2. Moments of the Gaussian prior distributions used for fitting the linear model.

\begin{tabular}{lccc}
\hline Parameter & Mean & Standard Deviation & Units \\
\hline$c_{m}$ & 170 & 50 & $\mathrm{Ns} / \mathrm{m}$ \\
$\sigma$ & 0.07 & 0.03 & - \\
\hline
\end{tabular}

Table 3. Mean posterior parameter estimates for the linear model (estimated using 1000 samples).

\begin{tabular}{lcc}
\hline Parameter & Posterior Mean & Units \\
\hline$c_{m}$ & 432.6 & $\mathrm{Ns} / \mathrm{m}$ \\
$\sigma$ & 0.1035 & - \\
\hline
\end{tabular}

distribution), an ensemble of model responses was generated. The mean response of this ensemble is compared with the training data in Figure 7, where the confidence bounds are three standard deviations from the mean. Clearly, the linear model is a poor representation of the real system; it is interesting to note that there are regions where the real device appears to be 'sticking' which the model is unable to replicate. Based on this observation as well as the damping-force plots shown by Cassidy et al. (2011), it was hypothesised that a hyperbolic tangent friction model may be a more appropriate choice.

\section{Hyperbolic tangent}

In this case a model of the form $f(\dot{z})=F_{c} \tanh (\beta \dot{z})$ was employed. The vector of parameters to be identified was therefore $\boldsymbol{\theta}=\left\{c_{m}, F_{c}, \beta, \sigma\right\}$. As before, Gaussian distributions truncated at zero were used as priors (moments are shown in Table 4).

The resulting samples from the posterior are shown in Figure 8 while the mean posterior parameter estimates are given in Table 5. It is encouraging to note that the mean values of $c_{m}$ and $F_{c}$ are fairly close to those that were estimated in 'Initial modelling'.

Finding that the model was able to replicate the training data well, its ability to predict $80 \mathrm{~s}$ of 'unseen' data - data which was not used to infer the parameter estimates - was investigated using Monte Carlo simulations. The average response of the model as well as the resulting confidence bounds are compared with experimental results in Figures 9 and 10: Figure 9 shows a small section of the model response while Figure 10 shows the full $80 \mathrm{~s}$. It is clear that the model is able to make accurate predictions about the response of the real system and, although $1.3 \%$ of the measured response fell outside of the confidence bounds (a possible solution to this issue is discussed in 'Discussion and future work'), there are no regions where the measured dynamics of the device are dramatically different from that predicted by the model. 


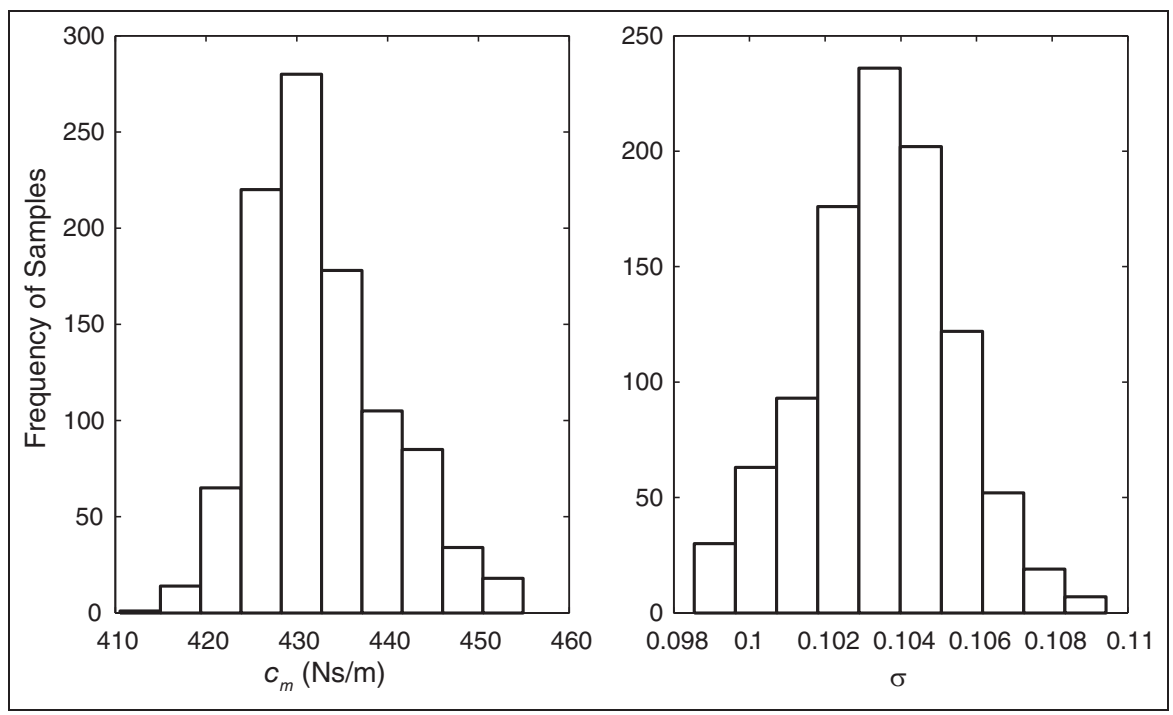

Figure 6. MCMC results for the linear model.

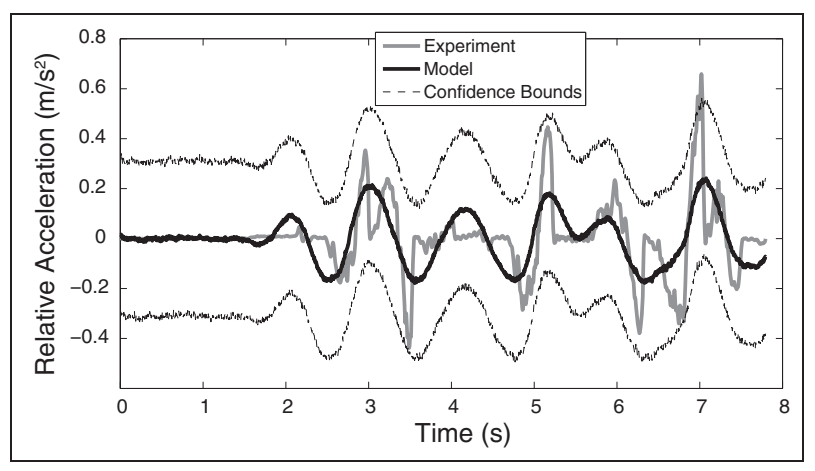

Figure 7. Comparison between the average response of the linear model (black) and the training data (grey).

Table 4. Moments of the Gaussian prior distributions used for fitting the hyperbolic tangent model.

\begin{tabular}{lccc}
\hline Parameter & Mean & Standard Deviation & Units \\
\hline$c_{m}$ & 170 & 50 & $\mathrm{Ns} / \mathrm{m}$ \\
$F_{c}$ & 8.4 & 5 & $\mathrm{~N}$ \\
$\beta$ & 100 & 100 & $\mathrm{~s} / \mathrm{m}$ \\
$\sigma$ & 0.07 & 0.03 & - \\
\hline
\end{tabular}

Having used TMCMC to estimate equation (11) for both model structures, their relative probabilities could then be assessed using equation (7). Writing the linear and hyperbolic tangent model structures as $M_{1}$ and $M_{2}$ respectively and assigning equal prior probabilities to each model, it was found that

$$
\log \left(\frac{P\left(M_{2} \mid D\right)}{P\left(M_{1} \mid D\right)}\right)=24.2
$$

Table 5. Mean posterior parameter estimates for the hyperbolic tangent model (estimated using 1000 samples).

\begin{tabular}{lcc}
\hline Parameter & Posterior Mean & Units \\
\hline$c_{m}$ & 109.5 & $\mathrm{Ns} / \mathrm{m}$ \\
$F_{c}$ & 11.86 & $\mathrm{~N}$ \\
$\beta$ & 88.35 & $\mathrm{~s} / \mathrm{m}$ \\
$\sigma$ & 0.0956 & - \\
\hline
\end{tabular}

This helps confirm the belief that, relative to the linear model, the hyperbolic tangent model is the most probable. The end result, then, is a probabilistic model of a rotational energy harvester that can be used to make predictions which, by using samples from the posterior distribution, are robust against the uncertainties one has about the physics of the real system.

\section{Discussion and future work}

The work presented here has been focused specifically on the modelling of the mechanical portion of a rotational energy harvester. With a robust model established, an obvious next step would be to develop a probabilistic model of the electrical portion of the device. This could be achieved through using the base acceleration as the model input and then using the voltage output of the real device to infer parameter estimates. Subsequently exciting the model with ambient acceleration data, it would then be possible to make predictions about how the energy harvester would perform in real scenarios - these predictions would be robust against uncertainties in both the mechanical and electrical components of the model. 


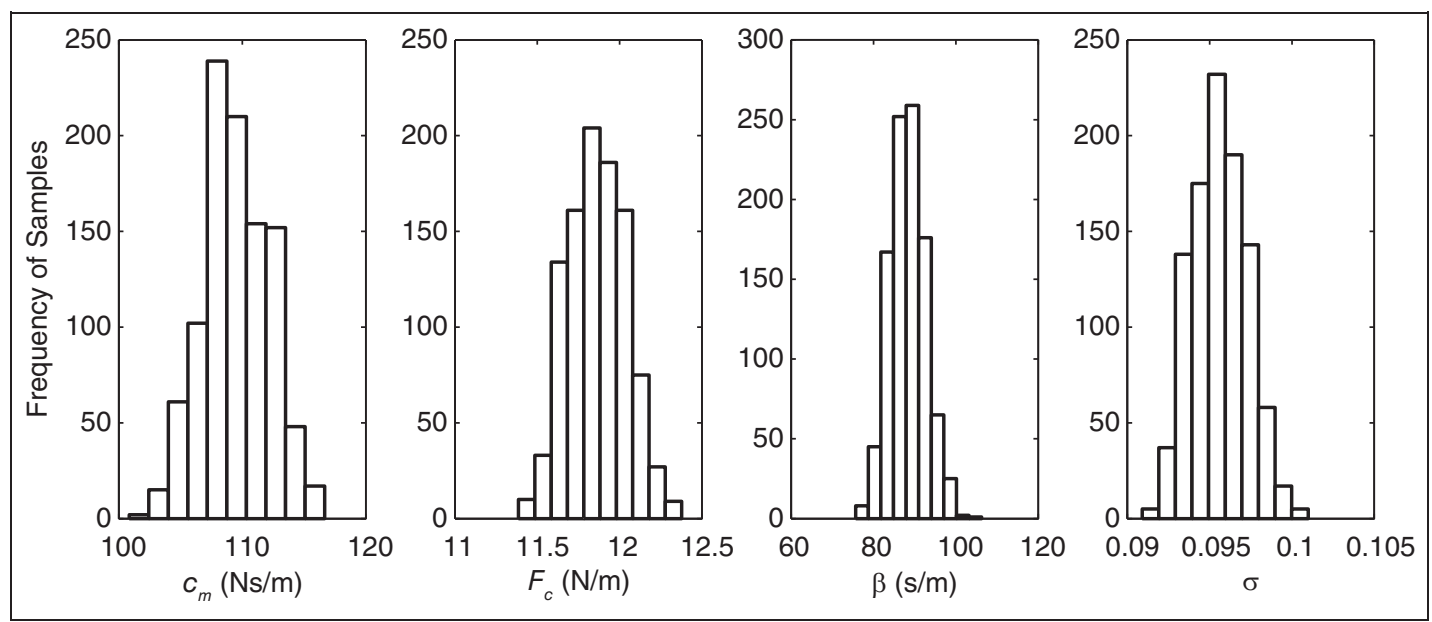

Figure 8. MCMC results for the hyperbolic tangent model.

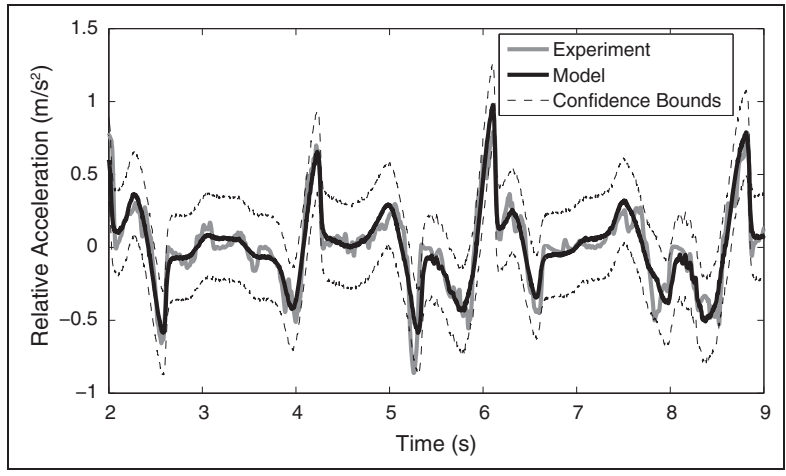

Figure 9. Comparison between the average response of the hyperbolic tangent model (black) and a small section of previously ‘unseen' experimental data (grey).
The authors plan to utilise the model developed in the current work to optimise this design of energy harvester, possibly using the work shown in Hendijanizadeh et al. (2014), such that its performance could be improved. The authors also intend to use a similar procedure to generate probabilistic models of inerter devices (which also utilise rack-and-pinion/ballscrew mechanisms) - this would be building on works such as Papageorgiou et al. (2009) where the modelling and testing of inerter devices was considered in detail. It is worth noting that, as an alternative to the rotational devices described here, there are also devices which utilise a 'plucking-based' method to achieve frequency up-conversion (Pozzi and Zhu, 2011) which are certainly also worthy of further investigation.

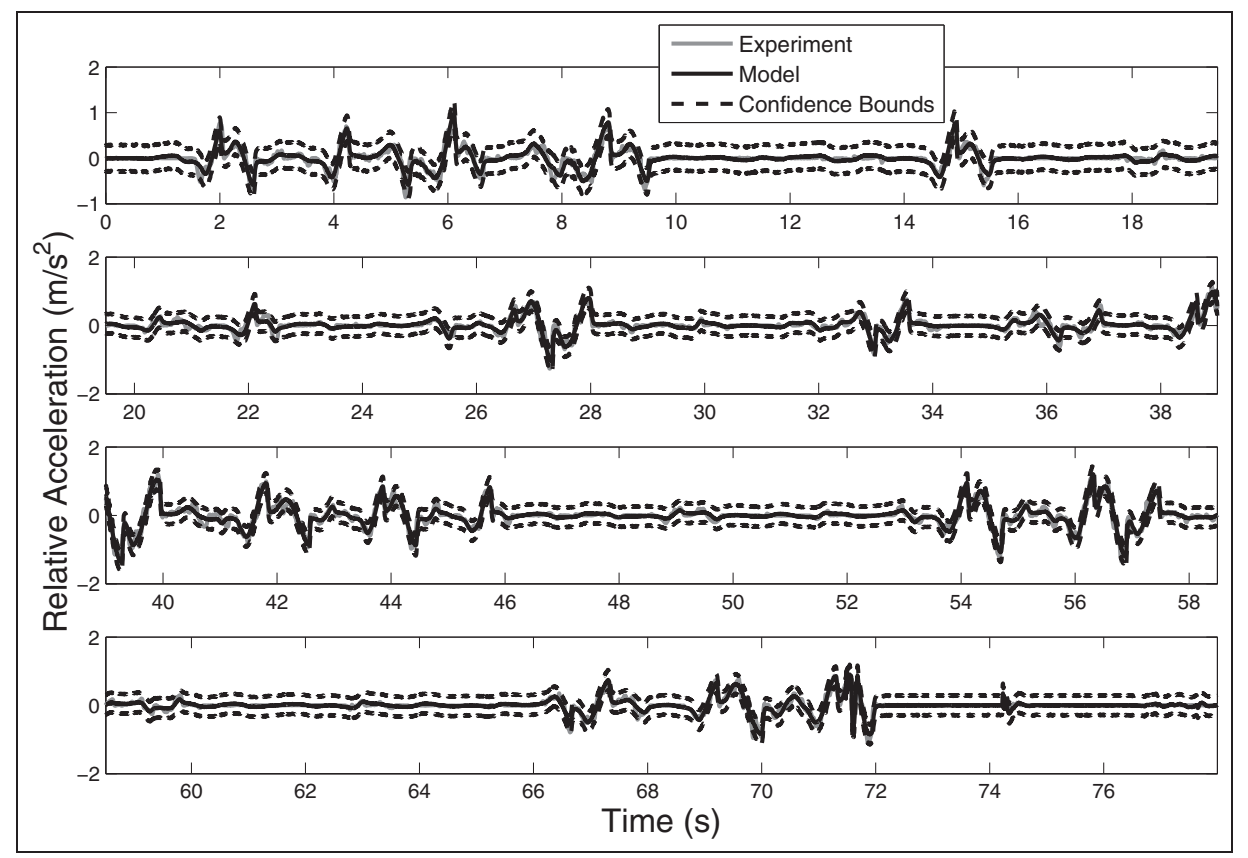

Figure 10. Comparison between the average response of the hyperbolic tangent model (black) and roughly $80 \mathrm{~s}$ of previously 'unseen' experimental data (grey). 
With regard to the hyperbolic tangent model, it was observed that the measured response fell outside of the confidence bounds more often than expected (although not by large amounts). It is conjectured here that this may be because the $80 \mathrm{~s}$ of data used for validation purposes may contain dynamics which was not present in the relatively small set of training data. An obvious solution to this issue would be to use larger sets of training data but, as numerical integration techniques were used in this case, such a strategy would greatly increase the computational cost of running MCMC. For future work the authors intend to address this issue using the methods presented by Green et al. (2014), where it was shown that small 'information rich' subsets can be extracted from large sets of training data and used to infer parameter estimates.

Finally, noting that some of the potential applications of rotational energy harvesters feature ambient excitations which can be approximated as white noise (the excitations which arise as a result of road roughness for example; Li et al., 2013), it may be advantageous to analyse the response of rotational energy harvesters to random excitations. This could draw on works such as Litak et al. (2010), Daqaq (2010), Green et al. (2012) and Langley (2014), where the response of nonlinear energy harvesters (including those which are designed to exhibit stochastic resonance) to white noise excitations has been extensively investigated.

\section{Conclusions}

This article is primarily concerned with the development of a robust, probabilistic model of a rotational energy harvester. Through the application of a Bayesian framework, a model of the device is inferred from a set of experimental data. It is then shown that the model is not only able to replicate nonlinearities in the real device (friction effects specifically), but is also able to make predictions which are robust against parameter uncertainties. For future work the authors aim to use this model to probabilistically analyse the ability of the device in question to harvest kinetic energy from high-amplitude, low-frequency excitations (such as those induced by ocean waves or the motion of human walking).

\section{Acknowledgement}

The authors would like to thank Dr Thibaut Putelat from the University of Bristol for his useful insights into friction modelling.

\section{Declaration of conflicting interests}

The authors declared no potential conflicts of interest with respect to the research, authorship, and/or publication of this article.

\section{Funding}

The authors would like to acknowledge the EPSRC Programme Grant 'Engineering Nonlinearity' (EP/K003836/ 1) which funded this collaborative project.

\section{Note}

1 It is important to note that by the term 'probabilistic model', the authors are referring specifically to a model whose structure is deterministic but whose parameters are known only probabilistically.

\section{References}

Agamloh EB, Wallace AK and von Jouanne A (2008) A novel direct-drive ocean wave energy extraction concept with contact-less force transmission system. Renewable Energy 33(3): 520-529.

Brown P, Hardisty D and Molteno TCA (2007) Wavepowered small-scale generation systems for ocean exploration. In: OCEANS 2006 - Asia Pacific, pp. 1-6.

Cassidy IL, Scruggs JT, Behrens S, et al. (2011) Design and experimental characterization of an electromagnetic transducer for large-scale vibratory energy harvesting applications. Journal of Intelligent Material Systems and Structures 22(17): 2009-2024.

Ching J and Chen YC (2007) Transitional Markov chain Monte Carlo method for Bayesian model updating, model class selection, and model averaging. Journal of Engineering Mechanics 133(7): 816-832.

Choi SB, Seong MS and Kim KS (2009) Vibration control of an electrorheological fluid-based suspension system with an energy regenerative mechanism. Proceedings of the Institution of Mechanical Engineers, Part D: Journal of Automobile Engineering 223(4): 459-469.

Daqaq MF (2010) Response of uni-modal Duffing-type harvesters to random forced excitations. Journal of Sound and Vibration 329(18): 3621-3631.

Doob JL (1953) Stochastic Processes (Wiley Publications in Statistics). New York, NY: Wiley.

Green PL, Cross EJ and Worden K (2015) Bayesian system identification of dynamical systems using highly informative training data. Mechanical Systems and Signal Processing. 56: 109-122.

Green PL, Papatheou E and Sims ND (2013) Energy harvesting from human motion and bridge vibrations: An evaluation of current nonlinear energy harvesting solutions. Journal of Intelligent Material Systems and Structures 24(12): 1494-1505.

Green PL, Worden K, Atallah K, et al. (2012) The benefits of Duffing-type nonlinearities and electrical optimisation of a mono-stable energy harvester under white Gaussian excitations. Journal of Sound and Vibration 331(20): 4504-4517.

Hendijanizadeh M (2014) Design and optimisation of constrained electromagnetic energy harvesting devices. $(\mathrm{PhD}$ thesis) The University of Southampton.

Hendijanizadeh M, Moshrefi-Torbati M and Sharkh SM (2014) Constrained design optimization of vibration energy harvesting devices. Journal of Vibration and Acoustics 136(2): 021001. 
Hendijanizadeh M, Sharkh SM, Elliott SJ, et al. (2013) Output power and efficiency of electromagnetic energy harvesting systems with constrained range of motion. Smart Materials and Structures 22(12): 125009.

Langley RS (2014) A general mass law for broadband energy harvesting. Journal of Sound and Vibration 333(3): 927-936.

Litak G, Friswell MI and Adhikari S (2010) Magnetopiezoelastic energy harvesting driven by random excitations. Applied Physics Letters 96(21): 214103.

Li Z, Zuo L, Luhrs G, et al. (2013) Electromagnetic energyharvesting shock absorbers: Design, modeling, and road tests. IEEE Transactions on Vehicular Technology 62(3): 1065-1074.

Matsuoka T, Omata K, Kanda H, et al. (2002) A study of wave energy conversion systems using ball screwsComparison of output characteristics of the fixed type and the floating type. In: Proceedings of the international conference on offshore and polar engineering, pp. 581-585.

Metropolis N, Rosenbluth AW, Rosenbluth MN, et al. (1953) Equation of state calculations by fast computing machines. The Journal of Chemical Physics 21(6): 1087-1092.

Muto M and Beck JL (2008) Bayesian updating and model class selection for hysteretic structural models using stochastic simulation. Journal of Vibration and Control 14(1-2): 7-34.
Papageorgiou C, Houghton NE and Smith MC (2009) Experimental testing and analysis of inerter devices. Journal of Dynamic Systems, Measurement, and Control 131(1): 011001.

Papatheou E and Sims ND (2012) Developing a hardware inthe-loop simulator for a backpack energy harvester. Journal of Intelligent Material Systems and Structures 23(7): $827-835$

Papatheou E, Green P, Racic V, et al. (2012) A short investigation of the effect of an energy harvesting backpack on the human gait. In: SPIE smart structures and materials, nondestructive evaluation and health monitoring.

Pozzi M and Zhu M (2011) Plucked piezoelectric bimorphs for knee-joint energy harvesting: Modelling and experimental validation. Smart Materials and Structures 20(5): 055007.

Rome LC, Flynn L, Goldman EM, et al. (2005) Generating electricity while walking with loads. Science 309(5741): 1725-1728.

Simeone L, Tehrani MG, Elliott SJ, et al. (2014) Nonlinear damping in an energy harvesting device. In: Proceedings of ISMA 2014, international conference on noise and vibration engineering, Leuven, Belgium.

Simoen E, Papadimitriou C and Lombaert G (2013) On prediction error correlation in Bayesian model updating. Journal of Sound and Vibration 332(18): 4136-4152. 